\title{
Tecnura
}

REVISIÓN DE TEMA

\section{Generación de mapas de ruido (industrial) desde sistemas de información geográfica. Un acercamiento desde la literatura}

\section{Generation of maps of noise (industrial) from geographic information systems. An approach from the literature}

\author{
Gustavo Andrés Romero Duque1, Jairo Acero Calderón², Marcela Jaimes Becerra³
}

Fecha de recepción: 28 de febrero de 2014

Fecha de aceptación: 15 de mayo de 2016

Cómo citar: Romero Duque, G., Calderón Acero, J., \& Jaimes Becerra, M.(2016). Generación de mapas de ruido (industrial) desde sistemas de información geográfica. Un acercamiento desde la literatura. Revista Tecnura, 20(49), 152-166. doi: 10.14483/udistrital.jour.tecnura.2016.3.a10

\section{RESUMEN}

Contexto: El ruido en el ámbito laboral, en todo tipo de actividades productivas, representa un peligro y realmente no se ha valorado en su real dimensión. Poco se ha visto que las partes interesadas hayan determinado la urgencia de manejar programas de control del ruido. Por lo tanto, las pérdidas resultantes de los tratamientos médicos y el ausentismo, representados en el cuidado de la salud y servicios sociales, resultan en costos ocultos relacionados con el trabajo que afectan directamente el producto interno bruto en cualquier país.

Método: Este artículo recopila diferentes estudios de caso en todo el mundo. Los estudios se dividieron para su revisión en los estudios generales sobre los efectos del ruido de la mano de obra y luego particularizado de acuerdo a los efectos del ruido industrial en la salud de los trabajadores. A un nivel de control, la evaluación y medición del ruido se define mediante el uso de herramientas tales como mapas de ruido y sus respectivas derivaciones, además de bases de datos espaciales.
Resultados: De acuerdo a la recopilación de información y el análisis de la misma observamos que a mediano plazo las economías van estar disminuidas en un porcentaje importante debido a las consecuencias generadas por la exposición al ruido. La información específica se encuentra en el desarrollo del artículo.

Conclusiones: Los datos aportados por los estudios de caso señalan la necesidad que Colombia, país que no es ajeno a este fenómeno, y que adicionalmente tiene la gran desventaja de no contar con estudios significativos en el campo del análisis de ruido, deberá fortalecer los estudios basados en datos espaciales como mecanismo de medición y control.

Financiamiento: Fundación universitaria Los Libertadores.

Palabras clave: Mapa de ruido, Ruido, Sistema de información geográfica.

\section{ABSTRACT}

The noise in the workplace, in all kinds of productive activities, represents a menace and it true has

1 Ingeniero de producción, candidato a magíster en Ingeniería Industrial. Docente tiempo completo Fundación Universitaria Los Libertadores. Bogotá, Colombia. Contacto: garomerod@libertadores.edu.co

2 Administrador industrial, especialista en Salud Ocupacional, candidato a magister en Gestión de Proyectos. Docente de tiempo completo Fundación Universitaria los Libertadores. Bogotá, Colombia. Contacto: jcalderona@libertadores.edu.co

3 Ingeniera de Sistemas, magíster en Ingeniería Área Informática y Ciencias de la Computación. Docente de tiempo completo Fundación Universitaria los Libertadores. Bogotá, Colombia. Contacto: bmjaimesb@libertadores.edu.co 
not been truly assessed in its real dimension. Little has been seen from the people concerned in this matter to give the urgency to manage noise control programs.

Therefore, disposals resulting from medical treatments and absenteeism, represented in health care and social services, result in labor-related hidden costs that directly affect the gross domestic product in any country.

Method: This article compiles different studies worldwide. The studies were divided for revision into general studies about the effects of labor noise and then, particularized according to the effects of industrial noise on workers' health. At a control level, the evaluation and measurement of noise is defined by using tools such as noise maps and their respective derivations in addition to spatial databases.

Conclusions: The data provided by the case studies points to the need in Colombia, a country that is no stranger to this phenomenon, and additionally has the great disadvantage of not having significant studies in the field of noise analysis.

It strengthens studies based on spatial data and measurement and control mechanism.

Keywords: Geography information system, Noise, Noise map.

\section{INTRODUCCIÓN}

El ruido, una sensación auditiva desagradable que con exposiciones prolongadas conlleva a una disminución progresiva de la capacidad auditiva, genera como consecuencia, disminución de la calidad de vida del individuo e impactos negativos en su productividad.

Se ha establecido que las personas que están expuestas al ruido a niveles superiores a 85 decibeles ponderados (en adelante dBA), sufren pérdida de la audición. Esta pérdida es de carácter neurosensorial, bilateral y simétrica, lo cual implica lesión en el interior del oído, por soportar frecuencias más altas (3k, 4k o $6 \mathrm{k} \mathrm{Hz}$ y y luego se extiende a las frecuencias más bajas $(0,5 k, 1 k, 2 k \mathrm{~Hz})$. (Subroto y Dhatrak, 2008).

El oído es un órgano muy sensible y al ser sometido a niveles altos de ruido puede sufrir modificaciones anatómicas hasta llegar a la pérdida definitiva de la audición. Esta patología tiene dos etapas: en la primera se presenta una hipoacusia que se alivia completamente después de un periodo de descanso y realiza cambios temporales en el umbral auditivo, y la segunda se produce cuando se sigue expuesto crónicamente al ruido y ocurren cambios permanentes, lo que ocasiona, por un lado, un daño irreversible en el órgano de Corti, además de la hipoacusia neurosensorial, evidente después de estar expuesto de 10-15 años al estímulo auditivo casual (Otárola, Otárola y Finkelstein, 2006).

Otro inconveniente que ocasiona el ruido es la generación de costos en la industria, debido a la pérdida de la productividad, la educación especial y los cuidados médicos que se ocasionan por la pérdida de la audición en los trabajadores (Díaz, 2006).

Por estas razones, las industrias deben establecer programas de vigilancia para monitorear a los trabajadores que están expuestos a niveles de ruido que impliquen un riesgo para su salud y que pueden adquirir sordera profesional (Sánchez y Albornoz, 2006).

En este artículo de revisión se consolidan diferentes estudios sobre los efectos del ruido laboral en la salud del individuo, así como sus controles, mediante herramientas para la gestión y evaluación del ruido, como los mapas industriales que permiten georreferenciar los resultados con diferentes metodologías y cálculos.

Las bases de datos, otro componente del artículo, indican cómo se constituye una infraestructura de datos espaciales aplicando, los geoprocesamientos, en interacción con los sistemas de información geográfico y los sistemas de posicionamiento geográfico. 
Actualmente en las empresas manufactureras se ha encontrado una importante incidencia negativa en la salud auditiva de los operarios y funcionarios que permanecen mayor tiempo en el área donde se localiza la planta de producción (Shigiang, Ruixiang, Kun y Yu, 2008) o donde se encuentren las máquinas.

\section{ESTUDIOS SOBRE LOS EFECTOS DEL RUIDO LABORAL}

Se han realizado muchos análisis sobre las consecuencias del ruido laboral sobre los trabajadores. Entre otros, se tienen:

En el estado de Aragua (Venezuela) se realizó un estudio con 122 trabajadores de la zona industrial de la ciudad La Victoria, de donde se obtuvo que el $30,4 \%$ de los trabajadores presentaron problemas auditivos. El 70,4\% de los trabajadores manifestó dificultades extraauditivas, siendo las más frecuentes, el insomnio en 49,1\%, la irritabilidad en el $40 \%$ y la cefalea en $31,9 \%$ de los casos. El 11,4\% refirió problemas en el área sexual; 19,1\% de los trabajadores expresaron ser hipertensos por la exposición laboral al ruido; en 33,5 \% de ellos se diagnosticó ansiedad, y en 5,8 \% depresión. El 27 \% recibió incapacidad de forma permanente y total (Martínez, 1995).

En Turquía, 126 trabajadores se vieron expuestos a altos niveles de ruido de hasta ocho horas diarias en una planta de energía hidroeléctrica. Aunque la empresa les había proporcionado protectores auditivos a los trabajadores, estos no los utilizaban. Se diagnosticó a 71 de los 126 trabajadores expuestos al ruido con deficiencia auditiva. Esta se generó durante los primeros de diez años de exposición, sin embargo, se detectó un mínimo de cambio de umbral permanente en un periodo de 40 años (Celik, Yalcın, y Öztürk, 1998).

En Chipre (Eleftheriou, 2002) se realizó un estudio a más de 90 empresas con una muestra de 200 personas y del cual se obtuvieron los siguientes resultados: 71,6\% de los trabajadores encuestados estuvieron expuestos a una dosis menor que la permitida para su turno, mientras que el resto $(28,4$ $\%$ era expuesto a una dosis más alta. De un total de 90 instalaciones industriales, 14 expusieron a sus trabajadores a dosis de ruido que superaban el valor límite en un $100 \%$. El 27,8 \% de los encuestados presentó un ligero cambio permanente del umbral entre 20 y $30 \mathrm{~dB}$, mientras que el $7,7 \%$ de los examinados tuvieron una grave discapacidad con un desplazamiento permanente del umbral superior a $30 \mathrm{~dB}$. El porcentaje de las personas que presentaron alguna discapacidad auditiva fue del 35,5\%.

En la ciudad de Sivas (Turquía) se investigó sobre el ruido generado por fábricas de hormigón poligonal, hierro y acero, cemento y textiles mediante un estudio que fue practicado a 256 trabajadores de estas industrias. El nivel de perturbaciones por ruido industrial que manifestaron los trabajadores se encontró en un 73,83\%. El porcentaje de trabajadores que, según la empresa, expresó problemas de audición fue: 30,86 \% en la empresa de concreto hormigón transversal; 33,33 \%, en la de cemento; $23,96 \%$, en la de hierro y acero, y en las fábricas textiles, $40 \%$. El 85,94\% de los trabajadores no tienen pruebas de audición periódica. Los accesorios de protección del oído se utilizan en las industrias con una tasa de 32,94\% (Atmaca, 2005).

Se realizó un estudio a 82 trabajadores de la fábrica de Productos Lácteos Escambray, de Cumanayagua (Cienfuegos, Cuba); la empresa está conformada por tres líneas de producción: fábrica de helados, de quesos y pasteurizadora. Fueron analizadas 24 áreas, 15 (62,5\%) tenían niveles de ruidos igual o superior a $85 \mathrm{dBA}$, y fue la pasteurizadora la zona que presentó mayor número de problemas de niveles de ruido elevado (85,7\%); la fábrica de quesos solo tuvo tres áreas $(42,9 \%$ ) con niveles superiores a $85 \mathrm{dBA}$. El estudio determinó que la empresa en un $100 \%$ de las áreas estudiadas, presentaba ruido continuo y no intermitente (Moreno, Martínez y Rivero, 2006).

Otro estudio fue realizado a 98 trabajadores de la Carpintería de Aluminio Tomás Álvarez Breto, situada en La Habana. La empresa fue dividida en 13 áreas de las cuales se comprobó que solamente 
cuatro presentaban niveles de ruido iguales o inferiores a $85 \mathrm{dBA}$, el resto presentó niveles altos de ruido. Se determinó que el $78 \%$ de los trabajadores tenían hipoacusia y solamente $22 \%$ obtuvieron resultados normales. En los puestos donde el personal era rotado no se presentaron problemas auditivos. Se determinó que la disminución de la audición se presentó en un mayor porcentaje en las mujeres (45,9\%) que en los hombres $(32,7 \%)$ (Hernández y González, 2008).

En Venezuela, en una industria papelera, se realizaron audiometrías a sus trabajadores y mediciones de la intensidad del ruido ambiental. Los trabajadores estaban expuestos a niveles superiores de 85 dBA; aquellos expuestos a ruido contaban con mecanismos de protección como tapones y orejeras, ya que según las políticas corporativas, pero no eran utilizados por ellos. La mayoría de los trabajadores de los grupos expuestos a ruido presentaron problemas de audición (Guevara et al., 2008).

En la empresa Raja Tren de pasajeros (Irán), se realizó un estudio donde se establecieron dos grupos (154 y 146 trabajadores) que en su actividad se exponían a niveles de ruido alto y bajo, respectivamente. Se les aplicó un test de sangre, con métodos enzimáticos para estimar triglicéridos (TG), colesterol total (CT) y los niveles de lipoproteínas de alta densidad (HDL). El análisis mostró que trabajar en un lugar con alta exposición al ruido y el aumento de las horas laborales por semana, hizo que se incrementará el TG sérico en el grupo de alta exposición. Los niveles de CT y HDL no presentaron un aumento significativo en ninguno de los dos grupos, con lo cual fue posible determinar que el ruido crónico aumenta la presión arterial sistólica, entre los trabajadores sobre todo de sexo masculino (Mehrdad, 2011).

En Egipto, en 15 diferentes industrias, se realizaron estudios sobre sus niveles de ruido (con sonómetros), Ilevándose a cabo tomas en intervalos de cada 10 minutos durante periodos de 8 horas diarias y a una altura de 1,2 $\mathrm{m}$ sobre el suelo. Se estableció que los trabajadores debían soportar niveles de ruido que superaban los 90 dBA así: industria de comida $71,4 \mathrm{dBA}$, bebidas $73,1 \mathrm{dBA}$, ropa 74,5 dBA, madera 80,7 dBA, muebles 83,6 dBA, servicio de lavandería de hospital 86,2 dBA, molino de trigo 87,4 dBA, establecimiento de impresión 89,3 dBA, central eléctrica 91,5 dBA, taller de trabajo de metal 92,6 dBA, mantenimiento de vehículos 93,1dBA, mantenimiento para trenes 94,7 dBA, fábrica de aluminio 97,2 dBA, fábrica de hierro 98,5 dBA y obras de remache 100,4 dBA. Por medio de este estudio también se logró establecer que zonas residenciales presentaban niveles de ruido de 91,5 dBA cuando lo permisible es de 60 dBA. Los niveles máximos de ruido permisibles en las zonas educativas son $50 \mathrm{dBA}$, mientras que el nivel de ruido medido era 89,3 dBA. Los niveles máximos de ruido permisibles en áreas industriales son 70 dBA, mientras que el ruido medido en esta área fue de 98,5 dBA (Sayed, 2011).

\section{EFECTOS QUE CAUSA EL RUIDO INDUSTRIAL EN LA SALUD DE LOS TRABAJADORES}

A través de diversos estudios se ha establecido que el oído humano se ve afectado cuando se somete a niveles elevados de ruido y que la pérdida de la audición puede ser producida por ruido, sustancias ototóxicas y trauma acústico. De estas, la más frecuente, pérdida auditiva por ruido, afecta considerablemente la calidad de vida y se establece como enfermedad profesional. En la actualidad es fácil diagnosticarla por medio de la PTA (audiometría de tonos bajos) y la implementación de la OAE (prueba de emisiones otoacústicas) con estas pruebas es la mejor forma de establecer la hipoacusia neurosensorial ocupacional en los trabajadores expuestos. Así, la prueba que presenta mayor sensibilidad para detección de daño coclear es la OAE o la PTA (Gómez, 2012).

El ruido además genera efectos no auditivos como dilatación de las pupilas, agitación respiratoria, aumento de la presión arterial, menor irrigación sanguínea, disminución de la secreción gástrica, aumento del colesterol, aumento de 
la glucosa en la sangre, insomnio, fatiga, estrés, depresión, irritabilidad, histeria, neurosis, aislamiento social y falta de deseo sexual (Sánchez y Albornoz, 2006) Estos deterioros se presentan de una manera más rápida durante los primeros diez años de la exposición al ruido, luego su progreso es más lento (Celik, Yalcın y Öztürk, 1998).

También, el ruido tiene efectos sobre la salud como (Chávez, 2006):

- Malestar: ya que interrumpe las actividades que se están realizando. Este se comienza a experimentar a partir de niveles de $50 \mathrm{dBA}$

- Interferencia con la comunicación: una conversación moderada donde el locutor se encuentra a un metro, el nivel de presión se establece entre $50 \mathrm{dBA}$ y $55 \mathrm{dBA}$. Hablando a gritos se puede alcanzar a 75 dBA u 80 dBA. Se establece que un ruido de fondo con niveles superiores a $40 \mathrm{dBA}$ provocará dificultades en la comunicación oral. A partir de $65 \mathrm{dBA}$ de ruido, la conversación se torna extremadamente difícil.

- Pérdida de atención, de concentración y rendimiento: el ruido interfiere con la percepción y esto ocasiona problemas en la realización de una labor.

- Trastornos en el sueño: el ruido puede ocasionar dificultad para conciliar el sueño, también puede producir que se altere la presión arterial, arritmia cardiaca y movimientos corporales.

- Pérdida de la capacidad auditiva.

- Estrés.

- Habituación al ruido: el organismo se habitúa al ruido a mediano o largo plazo.

En algunos países, como la India, la pérdida de la audición se determinó como enfermedad indemnizable desde 1948 bajo la Ley del Seguro Estatal de Empleados y la Ley de Indemnización por Accidentes del Trabajo (1923). Pero no fue sino en 1996 que se presentó el primer caso de compensación y cerca de 250 trabajadores solamente están recibiendo indemnización por pérdida de la audición (Subroto y Dhatrak, 2008).

\section{CONTROL DEL RUIDO}

Se ha podido establecer que es fácil identificar los niveles de ruido que son peligrosos y técnicamente se puede realizar su control por medio de la aplicación de tecnología comercial, remodelando o transformando el equipo/proceso (Suter, Naturaleza y efectos del ruido, 2001).

Así mismo, la pérdida de audición es totalmente prevenible, esto se puede lograr por medio de controles de ingeniería y que los empleadores proporcionen a sus trabajadores al menos dos medios de protección: a) elementos de protección personal (EPP), que son los controles más comunes y b) las decisiones administrativas, como la rotación de turnos periódicos y limitar la exposición al ruido cuando los niveles excedan los 85 dB (Gómez, 2012).

Para prevenir la pérdida de la audición se debe: disminuir la exposición al ruido, usar tapones en los oídos cuando se va a estar expuesto a periodos largos de ruido, usar materiales que absorban el ruido y lo disminuyan en la casa o en el trabajo, no utilizar varias máquinas ruidosas al mismo tiempo, y tratar de evitar oír el ruido indeseable con otros sonidos y realizar periódicamente un examen de audición para evaluar su capacidad auditiva (Gómez, 2012).

Un mecanismo de protección contra el ruido de los trabajadores son los protectores auditivos; cuando estos se van a seleccionar se debe tener en cuenta el personal que lo va a utilizar, la compatibilidad con otros equipos de seguridad y las condiciones de trabajo como temperatura, humedad y presión atmosférica (Subroto y Dhatrak, 2008).

Los países en desarrollo se han concentrado en el control del ruido por medio de normas tendientes a establecer como límite de exposición permisible 85 dBA y un factor de acumulación (relación de interdependencia tiempo/intensidad) de $3 \mathrm{~dB}$, y métodos técnicos, como la implementación de pruebas audiométricas periódicas, utilización de protectores auditivos, la formación y el mantenimiento de registros (Suter, 2001).

Cuando una empresa establece un programa de control de ruido, lo primero que debe realizar 
es determinar cuál es el ruido aceptable en su industria, de esta forma puede establecer si se debe construir una nueva planta, ampliar sus instalaciones o comprar nuevos equipos. Después debe distribuir los equipos y los procedimientos según el ruido que estos generen, buscando que los procesos/máquinas más ruidosos estén juntos y separados de las áreas más silenciosas por zonas de ruido intermedio (Driscoll, 2001).

El siguiente paso es determinar las fuentes del ruido. Existen algunas técnicas que contribuyen a identificar el origen o la fuente del ruido (Driscoll, 2001):

- Medir el espectro de frecuencias y representar los datos gráficamente.

- Medir el nivel sonoro, en dBA, en función del tiempo.

- Comparar los datos de frecuencias con equipos o líneas de producción similares.

- Aislar componentes con controles temporales o conectar y desconectar un equipo tras otro, siempre que sea posible.

El primer paso del proceso de control del ruido es tratar directamente la fuente. El ruido también se puede controlar mediante cerramientos y barreras acústicas, que bloqueen o protejan al receptor de la vía sonora directa. Otra manera de controlarlo es instalando garitas o cabinas insonorizadas y de esta forma se aísla al empleado del ruido excesivo. Igualmente, se deben crear turnos de trabajo para que el empleado pueda recuperarse del ruido (Driscoll, 2001).

\section{EVALUACIÓN Y MEDICIÓN DEL RUIDO}

En las industrias es necesario que se realicen mediciones de emisión de ruido de fuentes fijas, para esto se debe tener en cuenta las siguientes condiciones generales (Echeverri, 2011):

- Los datos deben ser obtenidos a través de un sonómetro clase 1 o clase 2 según la norma
IEC 61672-1:2002 o cualquiera que la sustituya. Estos deben ser calibrados según la norma IEC 60942:2003.

- Deben existir condiciones atmosféricas adecuadas, es decir, tiempo seco y los pavimentos y las superficies sobre las que se efectúen las mediciones deben estar libres de humedad.

- La Resolución 627 del 7 de abril de 2006, del Ministerio de Ambiente, Vivienda y Desarrollo Territorial, determina que los niveles de presión sonora medida se deben corregir por impulsividad, tonalidad, condiciones meteorológicas, horarios, tipos de fuentes y receptores.

Existen parámetros para la medición de la emisión de ruido proveniente de fuentes fijas, los principales son (Echeverri, 2011):

- Nivel de presión sonora continuo equivalente con filtro de ponderación A, LAeq,T y ponderación temporal rápida $(\mathrm{F})$.

- Nivel de ruido residual, LAeq, T, residual, medido como nivel de presión sonora continuo equivalente con filtro de ponderación A y ponderación temporal rápida $(F)$ o como nivel de permanencia L90.

El tiempo de medición es de una (1) hora y esta se puede realizar de forma continua o con intervalos no menores a 15 minutos, para capturar la información necesaria se deben realizar como mínimo 3 mediciones de 5 minutos cada una, para que los resultados sean representativos. Al realizar la medición se debe hacer en el día y cuando la emisión de ruido sea mayor. Se deben hacer 2 procesos de medición, uno con la(s) fuente(s) de emisión de ruido funcionando, durante el periodo de mayor emisión, para obtener el nivel de presión sonora continuo equivalente con filtro de ponderación $\mathrm{A}$, LAeq, $1 \mathrm{~h}$, y otro sin la(s) fuente(s) funcionando, para determinar el ruido residual, LAeq, 1h, residual (Echeverri, 2011).

Para la evaluación de la calidad acústica de recintos cerrados existen principalmente tres 
criterios: noise criteria curves (NC), room criteria curves (RC) y balanced noise criteria curves (NCB); sin embargo, es este último el que ha adquirido la mayor relevancia dado que considera un mayor rango de bandas de frecuencias. Estas curvas fueron creadas con el propósito de que el ruido de fondo no interfiera con el tipo de actividad que se desarrolla en un determinado recinto, permitiendo, además, que la comunicación entre las personas sea satisfactoria (Chávez, 2006).

El ruido es medido con instrumentos como: sonómetros, dosímetros y equipos auxiliares. Los sonómetros se clasifican por su precisión, desde el más preciso (tipo 0) hasta el más impreciso (tipo 3). El filtro más utilizado es la red de ponderación A. A los sonómetros modernos para que arrojen resultados más detallados se les conectan filtros de banda de octava y de tercio de banda de octava (Denisov y Suvorov, 2001).

Según la norma internacional (ISO 2204, 1979) determina que el ruido se puede medir según tres tipos de métodos:

a) De control: las zonas de trabajo son medidas con un sonómetro, utilizando un número limitado de puntos de medida.

b) De ingeniería: El nivel sonoro es medido a través del factor de ponderación $\mathrm{A}$. El número de puntos de medición y las gamas de frecuencias se determinan en función de los objetivos de medición. Se deben registrar los factores temporales.

c) De precisión: las mediciones globales del nivel sonoro se complementan con mediciones en banda de octava o de tercio de octava y se registran historiales de intervalos de tiempo apropiados en función de la duración y las fluctuaciones del ruido.

El ruido en el trabajo se puede medir bajo dos criterios básicos (Denisov y Suvorov, 2001):

- Se puede medir la exposición a la cual está sometido cada trabajador.

- Los niveles de ruido pueden medirse en varias áreas, realizando un mapa de ruido para determinar las áreas de riesgo.

\section{MAPAS DE RUIDO, HERRAMIENTA PARA LA GESTIÓN Y EVALUACIÓN DEL RUIDO}

La diversificación de las actividades humanas significa que un número creciente de ciudadanos está expuestos a distintas fuentes de ruido, a ambientes sonoros complejos (ruidos combinados).

Varios estudios han puesto de manifiesto el impacto del ruido en el bienestar y la salud humana (Pierrete et al., 2012) y definiendo que existe una relación causal entre el nivel sonoro y el grado de molestia que genera en las personas, aunque las características físicas y culturales del individuo pueden relativizar el nivel de impacto que tiene el ruido sobre la salud (Martínez y Moreno, 2005).

Desde hace tiempo la sociedad está concientizada en las implicaciones que tiene el ruido en el medio ambiental y en la salud. Como resultado, distintas entidades y personas han propuesto y desarrollado acciones encaminadas al control y a la reducción del ruido. Una de las herramientas más importantes para la evaluación y la gestión del ruido ambiental es la que se denomina mapa de ruido (Ayuntamiento de Leganés, 2011).

Los mapas de ruido se han utilizado para examinar los efectos de la contaminación acústica en sitios como aeropuertos, instalaciones de fábricas, rutas de carretera y comunidades residenciales cercanas. Su desarrollo ha permitido generar medidas correctivas como la rezonificación de áreas industriales/residenciales, así como la construcción de pantallas acústicas para reducir el efecto del ruido en alguna zona (Suebsak, Tawan y Supphawat, 1999).

Teniendo en cuenta lo trabajado por la Comunidad Europea en aspectos de evaluación, control y gestión del ruido (ambiental o industrial), se puede definir un mapa de ruido como:

[...] la presentación de datos sobre una situación acústica existente o pronosticada en función de un indicador de ruido, en la que se indicará el rebasamiento de cualquier valor límite pertinente vigente, 
el número de personas afectadas en una zona específica o el número de viviendas expuestas a determinados valores de un indicador de ruido en una zona específica (Parlamento Europeo, 2002).

Asimismo, define un mapa estratégico de ruido como "un mapa diseñado para poder evaluar globalmente la exposición al ruido en una zona determinada, debido a la existencia de distintas fuentes de ruido, o para poder realizar predicciones globales para dicha zona" (Parlamento Europeo, 2002).

El desarrollo de los mapas de ruido puede generarse de forma experimental o bajo cálculo matemático. Los mapas que se realizan de forma experimental suponen una mayor necesidad de recursos (humanos y económicos) iniciales, siendo lentos de realizar y su utilidad se alcanza en la etapa de diagnóstico, por llevar datos tomados in situ, lo que le da fiabilidad a la información (Simón et al., 2008).

Para la vigilancia del medio ambiente (en este caso el factor ruido) el aspecto de datos es de particular relevancia. De hecho, hoy existe mucha demanda por parte de los ciudadanos, grupos de activistas e incluso administraciones municipales para el uso de tecnologías participativas para hacer frente a la contaminación (D'Hondt, Stevens y Jacobs, 2013), las cuales si se aplica correctamente, pueden lograr la misma precisión que las técnicas estándar de mapas de ruido (D'Hondt, Stevens y Jacobs, 2013).

De igual modo, el desarrollo de los mapas de calculados presenta una mayor flexibilidad (se puede mapear mayor área de estudio), son económicamente más asequibles y su actualización es más fácil. Sus defectos radican en el hecho de que sus resultados dependen del modelo matemático utilizado y en el conjunto de datos de entrada que se introducen para su cálculo (Simón et al., 2008).

En principio, un mapa de ruido consiste en una representación espacial de datos sobre una situación acústica existente o pronosticada en función de un índice de ruido. En el caso más general, representa los niveles sonoros mediante un conjunto de líneas isófonas realizados a partir del cálculo de niveles sonoros en puntos receptores que abarcan toda la zona de estudio. Sin embargo, los mapas de ruido también pueden representar la superación de algún valor límite, el número de personas afectadas o el número de viviendas expuestas a un determinado valor del índice de ruido (Ayuntamiento de Leganés, 2011).

Conviene recordar que la cartografía acústica presenta muchas más posibilidades: mapas verticales, mapas con información numérica en puntos receptores, mapas de análisis de contribución de las distintas fuentes, mapas con población expuesta, etc. (Segués, 2005).

\section{MAPAS DE RUIDO INDUSTRIAL}

El desarrollo de mapas de ruido en las últimas dos décadas ha sido ampliamente trabajado, la mayoría de las grandes ciudades cuentan con departamentos técnicos de control de la contaminación acústica que elaboran habitualmente mapas de ruido y planes de actuación; es decir, existen ya procedimientos y una experiencia importante para la gestión del ruido ambiental (Segués, 2005).

Sin embargo, en un entorno más pequeño, como una oficina de negocios o un piso de una fábrica, el uso de un mapa de ruido es todavía limitado, en parte debido a la dificultad en la construcción de curvas de ruido (Suebsak, Tawan y Supphawat, 1999).

Un mapa de ruido industrial busca medir los niveles de ruido en empresas o fábricas industriales, tanto a nivel interno como el impacto a su alrededor. Busca, también, definir las emisiones generadas por las fuentes fijas que se encuentran en el interior de las empresas, para ello, es necesario determinar la distribución de la fábrica y en ella la ubicación de las máquinas. Una vez que se conocen los niveles de ruido en todos los puntos de interés, las curvas de ruido se pueden construir (Suebsak, Tawan y Supphawat, 1999). 


\section{METODOLOGÍA DE ELABORACIÓN DE MAPAS}

Antes de empezar a trabajar un mapa de ruido, es necesario establecer los requisitos y determinantes de dicho mapa; saber si se realizará un mapa general o específico sobre la(s) fuente(s), la escala de trabajo y precisión de los datos, los índices acústicos y resultados del mapa (Segués, 2005).

Luego se selecciona la técnica que se va a emplear (Segués, 2005): medidas con sonómetros (Icontec, 1992), métodos de previsión, o combinación de medidas y previsiones

Los mapas desarrollados bajo técnicas de medida directa (sonómetros) resultan costosos (recursos y tiempo), pero sus resultados reflejan un buen nivel de precisión. Los que se desarrollan con métodos de previsión presentan ventajas prácticas con buena fiabilidad de sus resultados, pero muchas veces establecer el comportamiento de las fuentes de ruido (cálculos) requiere un muy buen nivel de manejo de modelos matemáticos (Segués, 2005).

Los primeros mapas de ruido realizados en los años ochenta y noventa, se elaboraron con medidas experimentales (Recuero, Gil y Grundman, 1996) y es más adelante donde se empieza a componer una correcta elaboración a través de un plan de muestreo (Sommerhoff, Recuero y Suárez, 2004).

Las metodologías de muestreo espacial con medidas experimentales más empleadas para realizar mapas de ruido son (Ausejo, 2010): a) metodología de retícula o rejilla: dividiendo la zona bajo estudio mediante una rejilla de una distancia fija y realizar las medidas en los nodos de la rejilla (Recuero, Gil y Grundman, 1996) b) metodología de viales o tráfico: realizando una categorización de las vías y muestrear diferentes puntos de las vías asumiendo que las de la misma categoría presentarán niveles de ruido similares (Romeu y Jiménez, 2006); c) muestreo en zonas específicas: se suele emplear cuando se desea cubrir una zona específica en la que se centraliza un ruido específico, como el del ocio nocturno; d) metodología en función de los usos del suelo: se realiza teniendo en cuenta las características del suelo, en función de las categorías de planificación territorial de la aglomeración: uso comercial, uso residencial, etc. (Romeu y Jiménez, 2006); e) metodología de las zonas aleatorias: es habitual emplear esta metodología cuando no se puede trazar una retícula, no procede la utilización del método de viales o porque no hay una zona específica en la que se concentre el ruido (Pavón y Recuero, 2006).

\section{MÉTODOS DE CÁLCULO PARA MAPAS DE RUIDO}

El desarrollo del método de cálculo de focos industriales/focos puntuales (Gobierno Vasco, 2005) es uno de los más utilizados y estipula considerar las normas ISO que aportan el desarrollo del cálculo de las emisiones de fuentes puntuales (ISO $3740,2000)$ y su cálculo de propagación (ISO 9613, 1993), así como la determinación de emisiones de áreas industriales.

En Bogotá, la legislación aplicable a la problemática de ruido en la ciudad (SDMA, 2013), es entre otras, la norma nacional de emisión de ruido y ruido ambiente (Resolución 627/06 MAVDT) y la que establece la clasificación empresarial por impacto sonoro UCR que permite valorar las industrias y establecimientos, respecto a su nivel de generación de ruido (Resolución DAMA 832/00).

Así, se deben definir focos puntuales y sobre los mismos (día, tarde y noche) medir el nivel de potencia, describiendo la geometría del foco, su ubicación y direccionalidad. Para lo anterior se debe tener en cuenta que medir la presión sonora cercana al foco conlleva determinar los niveles de potencia de sonido con métodos de ingeniería (ISO 3744, 2010) y métodos de inspección (ISO 3746, 2010).

\section{BASES DE DATOS ESPACIALES}

Desde las primeras civilizaciones se vio la necesidad de representar de manera pictórica los datos espaciales. En el siglo XVIII en Europa se constituyó la Geographical Information Society a partir 
del establecimiento de los organismos de gobierno nacional con objeto de producir mapas catastrales y topográficos de todos los países. Estos institutos continúan vigentes. Incluso, a través de los dos últimos siglos los mapas se habían elaborado en estilos individuales; sin embargo, aún existen estándares dentro de la cartografía que no se han roto (Peña, 2010).

Las bases de datos espaciales surgen de la necesidad de almacenar y representar datos geográficos. Estas se constituyen en una base de datos electrónica que además de registrar y facilitar la administración de información trascendental de un dominio de negocio en un medio digital, permiten almacenar información de tipo espacial (Álvarez, 2009).

Una ventaja especial que proporciona la estructuración digital de la información es el análisis geoestadístico; la mayoría de software especializados en los sistemas de información geográfica (SIG), como el ArcGis, Quamtun Gis o el GrSig, presentan módulos para el análisis exploratorio de los datos espaciales y herramientas para crear superficies estadísticas (Miranda, Ubaque y Pinzón, 2015).

\section{INFRAESTRUCTURA DE DATOS ESPACIALES (IDE)}

El Instituto Geográfico Agustín Codazzi (IGAC) se mantiene como la entidad rectora de la geografía colombiana y de acuerdo con su misión, en su plan de desarrollo se definen las siguientes funciones (Arias, 2006):

- Incrementar el conocimiento y entendimiento de la visión, los conceptos y beneficios de las IDE.

- Evaluar e implementar el uso de metodologías comunes para la producción y mantenimiento de los datos fundamentales de soporte a las IDE.

- Contribuir con la generación de estrategias de cooperación entre el IGAC y otras organizaciones del orden nacional e internacional para promover y difundir el desarrollo de las IDE.

- Contribuir con el desarrollo de soluciones comunes para el descubrimiento, acceso y uso de los datos geoespaciales como respuesta a las necesidades de las comunidades.

El IGAC (2001) -como coordinador de la Infraestructura Colombiana de Datos Espaciales (ICDE), la cual es la suma de políticas, estándares, organizaciones y recursos tecnológicos que facilitan la producción, el acceso y el uso de la información geográfica de cubrimiento nacional, para apoyar el desarrollo económico y social del país- busca, entre otros, objetivos documentar los datos cartográficos básicos y temáticos producidos y facilitar el acceso a ellos (metadatos) y armonizar los sistemas de información para asegurar la interoperabilidad de los datos (estándares -comité de normalización de datos espaciales-) mediante la implementación de la a Norma Técnica Nacional de Metadatos Geográficos (NTC4611) (IGAC, 2000).

La columna vertebral de la IDE y de los sistemas cartográficos son los datos espaciales (figura 1). Los diversos marcos que componen para el

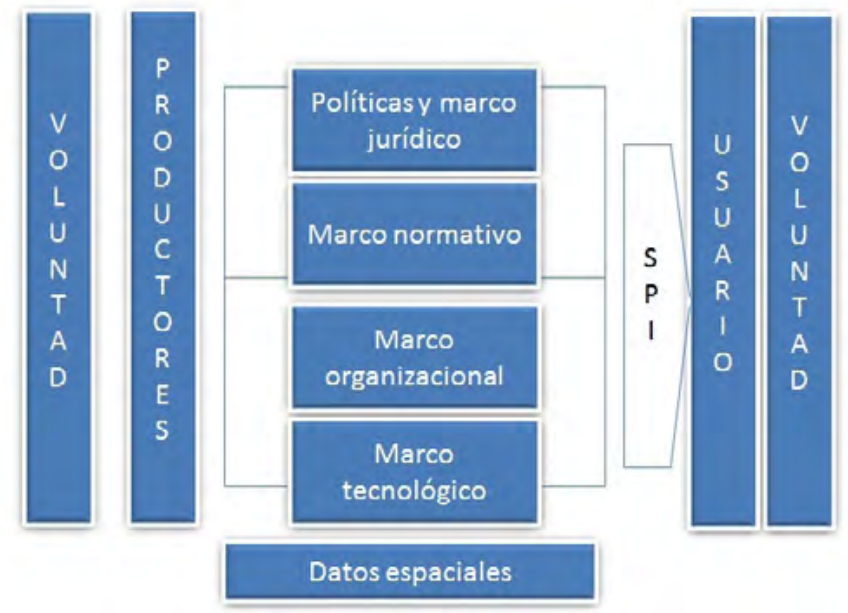

Figura 1. Modelo conceptual de la IDEMex.

Fuente: Esparza (2006). 
caso, la Infraestructura de Datos Mexicanos (IDEMex), están orientados hacia los datos espaciales. La IDEMex es el conjunto de políticas, marco jurídico, organizacional, normativo y tecnológico, necesarios para la efectiva creación e integración de datos espaciales y que faciliten la disponibilidad, acceso, compartición y aprovechamiento de estos, como parte del Sistema Público de Información (SPI) (Esparza, 2006).

\section{GEOPROCESAMIENTO APLICADO}

Eficientes herramientas para extraer información de datos espaciales son cruciales para organizaciones que toman decisiones basadas en conjuntos robustos de datos espaciales, como: la NASA (Administración Nacional de la Aeronáutica y del Espacio), la NIMA (Agencia Nacional de Imágenes y Cartografía) y la NSI (Instituto Nacional del Cáncer). Estas organizaciones están repartidas a través de muchos dominios de aplicación, incluyendo: ecología, administración del medio ambiente, seguridad pública, transporte, ciencias de la Tierra, epidemiología y la climatología (Ravikumar y Gnanabaskaran, 2010).

Teniendo en cuenta estudios tanto sociales como económicos, y en especial en los modelos econométricos, es necesario considerar las variables espaciales que se encuentran en el trabajo con datos georreferenciados; ya que los sucesos que ocurren en una ubicación tienen influencia en otra que aparentemente es remota (Acevedo y Velásquez, 2008).

Ejemplo de eso es el proyecto de Pacheco (2012), el cual obtuvo el índice de erosión potencial a través del tratamiento de datos edáficos, pluviométricos y térmicos, generando imágenes en mapas de tipo ráster, con una resolución espacial de 100 metros a través de interpolación .

Es recomendable una clase de mecanismo de despliegue de datos meteorológicos sobre la base del modo G/S el cual se basa en HGML (hyper geographic markup language) que es un estándar de intercambio uniforme. Para el caso de los datos meteorológicos que se caracterizan por ser densos y complicados. De acuerdo con el concepto de "concurrencia de la información" y "el servicio de polimerización", los cuales agrupa la tecnología de procesamiento geográfico ArcGIS (Chen, Miao, Yang y Xirong, 2011).

La tecnología de SIG se utilizó para integrar datos de la malaria en Colombia, de diversas fuentes para un período reciente y hacer distintos análisis espaciales en las cinco zonas endémicas identificadas. La base de datos espacial desarrollada permitió el ingreso periódico de datos para el seguimiento de la enfermedad y así evaluar oportunamente la efectividad de las estrategias adoptadas para la disminución de la incidencia (Molina, 2008).

\section{INTEROPERABILIDAD EN LOS SIG}

Considerando las dificultades en la interoperabilidad entre los archivos que generan los SIG, y que luego se pretendan manejar a través de otro SIG, es posible resolver empleando el formato " $\mathrm{dxf}$ ", pero a costa de pérdida de información y es allí donde surge la necesidad en la estandarización de la geo información (Bernabé, Manso y González, 2007).

Debido a que los datos espaciales son salvados en formato binario, representa un inconveniente para el uso y para compartir los datos. Con objeto de unificar la administración de los datos espaciales se propone mapear datos basado en XML (lenguaje de marcas extensible) en la base de datos relacional-espacial; esto, además de resolver los dos inconvenientes mencionados, proporciona la manera de acceder sistemas heterogéneos y como cualquier Web GIS garantiza el radio de uso de la información (Ye, Zang y Li, 2011).

\section{SISTEMA DE POSICIONAMIENTO GEOGRÁFICO (GPS)}

En contraste con el posicionamiento de vehículos, la localización de individuos con precisión está cobrando importancia, en especial en casos de 
salud, como es el caso de pacientes con demencia, entre otros, en donde se recomienda la arquitectura Mobile Guardian que se basa en un GPS y en GSM (management system geographic) (Wu, Yang, Liu y Dong, 2008).

Como proyecto estratégico para el desarrollo de Colombia, se estableció un convenio de cooperación entre el Departamento Administrativo Nacional de Estadística (DANE) y el IGAC para generar los productos cartográficos de soporte a los operativos censales, así como la cartografía básica. El IGAC ha avanzado en el establecimiento del Marco Geocéntrico Nacional de Referencia MAGNA y el uso de tecnologías GPS (global positioning system), para suministrar el sistema de coordenadas de precisión al cual se vinculan los diferentes tipos de cartografía para el censo y otros proyectos (Arias, 2006).

\section{CONCLUSIONES}

El uso de los SIG cada vez es mucho más amplio para muchos dominios de aplicación, esto sumado a la globalización y la proliferación de las redes, lleva a una necesidad de estandarizar las herramientas de software empleadas; es de anotar que en los años más resientes la mayoría de entidades orientadas a la cartografía digital usan la tecnología ESRI.

Las industrias en el mundo, por lo general, presentan ruido por encima de 85 decibeles, causando enfermedades auditivas irreversibles y otras asociadas al ruido, como: insomnio, irritabilidad, cefalea, problemas sexuales, hipertensión entre otras a mediano y largo plazo de exposición y al sistema productivo, pérdidas que aún no se cuantifican.

Por otra parte es importante destacar la importancia de investigar acerca del ruido ocupacional luego de conocer que el industrial puede generar afecciones, siendo entre otras la más grave, la perforación de manera permanente de la membrana timpánica del oído.
Las empresas en algunos sectores industriales, como las de metalmecánica, textil, alimentaria, plásticos, artes gráficas, entre otras, que son altamente generadoras de ruido, carecen por lo general de programas que lo controlen y, por ende, hay una falencia de una cultura de autocuidado, con lo cual se evidencia poco conocimiento y manejo acerca de este tema.

\section{REFERENCIAS BIBLIOGRÁFICAS}

Acevedo B., I. y Velásquez C., E. (2008). Algunos conceptos de la econometría espacial y el análisis exploratorio de datos espaciales. Ecos de Economía, 27, 28-30.

Álvarez P., V.L. (2009). Bases de datos espacio-temporales. México: Universidad Veracruzana.

Arias Duarte, L.P. (2001). El Instituto Geográfico Agustín Codazzi y su gestión como entidad líder de América en la construcción de infraestructura de datos espaciales (IDE). Revista Cartográfica, 73, 63-76.

Arias D., L.P. (2006). La cartografía digital del Censo General 2005 como estrategia para materializar resultados de la infraestructura colombiana de datos espaciales. Revista Cartográfica, 82, 157-168.

Atmaca, E.P. (2005). Industrial Noise and Its Effects on Humans. Journal of Enviromental Studies, 14(6), 721-726.

Ausejo, P.M. (19 de abril de 2010). Mapas de ruido. Recuperado el 15 de julio de 2013, de: http://www. aislacustic.com/servicios/mapas-de-ruido

Ayuntamiento de Leganés (2011). Mapa estratégico de ruido de la ciudad de Leganés. Leganés: Universidad Carlos III de Madrid.

Bernabé, M.A.; Manso, M.A. y González, M.E. (2007). La docencia universitaria sobre infraestructura de datos espaciales. Revista Cartográfica, 83, 17-35.

Celik, O.Y.; Yalcin, S y Öztürk, A. (1998). Noise parameters in industrial workers exposed. Auris Nasus Larynx, 25(4) 369-375.

Chávez M., J.R. (2006). Ruido: efectos sobre la salud y criterio de su evaluación al interior de recintos. Ciencia \& Trabajo, 8(20), 42-46. 
Chen, K.; Miao, F.; Yang, W. y Xirong, G. (2011). Research on meteorological data display based on $\mathrm{G} / \mathrm{S}$ model. IEEE, 683-686.

Denisov, E.I. y Suvorov, G. (2001). Medición del ruido y evaluación de la exposición. En J. M. Stellman, M. McCann y L. Warshaw. Enciclopedia de salud y seguridad en el trabajo (p. 47.6-47.8). Organización Internacional del Trabajo.

D'Hondt, E.; Stevens, M. y Jacobs, A. (02 de octubre de 2013). Participatory noise mapping works! An evaluation of participatory sensing as an alternative to standard techniques for environmental monitoring. Pervasive and Mobile Computing, 9(5), 681-694.

Díaz A., J. (2006). El ruido en el trabajo: alcance de un problema global. (39). Recuperado el 21 de noviembre de 2013, de: http://www.construmatica. com/construpedia/EI_Ruido_en_el_Trabajo._Alcance_de_un_Problema_Global

Driscoll, D.P. (2001). Técnicas de control del ruido. En: J.M. Stellman, M. McCann y L. Warshaw. Enciclopedia de salud y seguridad en el trabajo (pp. 47.847.12). Organización Internacional del Trabajo.

Echeverri L., C.A. (2011). Protocolo para medir la emisión de ruido generado por fuentes fijas. Revista Ingenierías, 10(18), 51-60.

Eleftheriou, P.C. (2002). Industrial noise and its effects on human hearing. Applied Acoustics, 63(1) 35-42.

Esparza R., L.G. (2006). Los datos, fundamento de una Infraestructura de Datos Espaciales. La experiencia mexicana. Revista Cartográfica, 82, 9-18.

Gobierno Vasco (1 de mayo de 2005). Sistema de consulta, información y gestión de focos de ruido ambiental. Guía metodológica para la realización de mapas de ruido. Comunidad Autónoma Vasca, España: Departamento de Ordenación del Territorio y Medio Ambiente.

Gómez M., M.J. (2012). Ruido industrial: efectos en la salud de los trabajadores expuestos. Revista CES Salud Pública, 3(2), 174-183.

Guevara, H.; Lugo, F.; Cardozo, R.; González, S.; Ortunío, M.; Sánchez, C. y Rivero, E. (2008). Exposición a ruido, solventes orgánicos y capacidad auditiva de trabajadores de una empresa papelera. Revista Informe Médico, 10(3), 149-158.
Hernández, D.A. y González, M.B. (2008) Alteraciones auditivas en trabajadores expuestos al ruido industrial. Medicina Seguridad Trabajo, 53(208), 1-11.

Instituto Colombiano de Normas Técnicas (Icontec) (1992). NTC 3428, Sonómetros-Medidores de la intensidad de sonido, 2-22.

Instituto Geográfico Agustín Codazzi. (2000). Acta de Acuerdo ICDE No. 1.

Instituto Geográfico Agustín Codazzi. (2001). Infraestructura Colombiana de Datos Espaciales (ICDE). Cartagena de Indias.

ISO 2204. (1979). International Organization for Standardization. Recuperado el 17 de septiembre de 2013, de: http://www.iso.org/iso/catalogue_detail. $\mathrm{htm}$ ?csnumber $=7007$.

ISO 3740 (2000). Acoustics - Determination of sound power levels of noise sources - Guidelines for the use of basic standards. International Organization for Standardization. Recuperado el 20 de 07 de 2013, de http://www.iso.org/iso/home/store/catalogue_tc/catalogue_detail.htm?csnumber $=1260$

ISO 3744. (2010). Acoustics - Determination of sound power levels and sound energy levels of noise sources using sound pressure - Engineering methods for an essentially free field over a reflecting plane. International Organization for Standardization. Recuperado el 19 de octubre de 2013, de: http:// www.iso.org/iso/iso_catalogue/catalogue_tc/catalogue_detail.htm?csnumber $=52055$

ISO 3746. (2010). Acoustics - Determination of sound power levels and sound energy levels of noise sources using sound pressure - Survey method using an enveloping measurement surface over a reflecting plane. International Organization for Standardization. Recuperado el 27 de octubre de 2013, de: http://www.iso.org/iso/home/store/catalogue_tc/catalogue_detail.htm?csnumber $=52056$

ISO 9613. (1993). Acoustics - Attenuation of sound during propagation outdoors - Part 1: Calculation of the absorption of sound by the atmosphere. International Organizational for Standardization. Recuperado el 24 de julio de 2013, de: http://www. iso.org/iso/iso_catalogue/catalogue_tc/catalogue_ detail.htm?csnumber $=17426$ 
Martínez S. y Moreno.J. (2005). Análisis espacio-temporal con SIG del ruido ambiental urbano en Madrid y sus distritos. Revista Internacional de Ciencia y Tecnología de la Información Geográfica, 5, 219-249.

Martínez, M.D. (1995). Efectos del ruido por exposición laboral. Salud de los Trabajadores, 3(2), 93-101.

Mehrdad, R.M. (2011). Relationship between Exposure to Industrial Noise and Serum Lipid Profile. Acta Medica Iranica, 49(11), 725-729.

Miranda, J.P.; Ubaque, C.A. y Pinzón, J.P. (2015). Selección de tecnologías para el tratamiento de aguas residuales municipales. Tecnura, 149- 164.

Molina, A.M. (2008). Sistema de Información Geográfica para el Análisis de la Distribución Geográfica de la Malaria. EIA, 9, 91-111.

Moreno, R.R.; Martínez, D.A. y Rivero, P.D. (2006). Pesquisa auditiva en trabajadores expuestos al ruido industrial. Revista Cubana Medicina General Integrada, 22(3).

Otárola, M.F.; Otárola, Z.F. y Finkelstein, K. A. (2006). Ruido laboral y su impacto en salud. Ciencia \& Trabajo, 8(20), 47-51.

Pacheco G., A. (2012). El índice de erosión potencial en la vertiente norte del Waraira Repano. Revista Colombiana de Geografía, 21(2), 85-97.

Parlamento Europeo (18 de julio de 2002). Diario oficial de las comunidades europeas. Recuperado el 21 de 11 de 2013, de: http:// eur-lex.europa.eu/LexUriServ/LexUriServ. do?uri=OJ:L:2002:189:0012:0012:ES:PDF

Pavón, I. y Recuero, M. (2006). Noise maps: a tool for the demarcation of risk areas of noise exposure in the surface mining industry. Ponencia en la ICSV13 The Thirteenth International Congress on Sound and Vibration. Viena.

Peña L., J. (2010). Sistemas de gestión geográfica aplicados a la gestión del territorio. San Vicente (Alicante): Editorial Club Universitario.

Pierrette, M.; Marquis-Favre, C.; Morel, J.; Rioux, L.; Vallet, M.; Viollon, S. y Moch, A. (17 de enero de 2012). Noise annoyance from industrial and road traffic combined noises: A survey and a total annoyance model comparison. Journal of Environmental
Psychology and a total annoyance model comparison, 32(2), 178-186.

Ravikumar, K. y Gnanabaskaran, A. (2010). ACO based spatial Data Mining for Traffic Risk Analysis. IEEE. International Conference on Innovative Computing Technologies, 1-6.

Recuero, M.; Gil, C. y Grundman, J. (1996). Mapa de ruido de San Sebastián de los Reyes. Metodología de medidas y resultados. Revista de Acústica, Número extraordinario, 51-54.

Romeu, J. y Jiménez, S. (2006). Spatial sampling for night levels estimation in urban environments. Journal of the Acoustical Society of America, 120(2), 791-800.

Sánchez, V.M. y Albornoz, V.C. (2006). Estrategia frente a la problemática del ruido ocupacional. Ciencia \& Trabajo, 8(20), 58-64.

Sayed A., A. (2011). Levels of industrial noise and annoyance in Egypt. Applied Acoustics, 72(4),221-225.

Secretaría Distrital del Medio Ambiente (SDMA) (2013). Información general sobre la problemática de ruido. Recuperado el 17 de octubre de 2013, de: http://ambientebogota.gov.co/ruido

Segués, E.F. (23 de marzo de 2005). Estrategia de elaboración de un mapa de ruido. Centro de Estudios y Experimentación de Obras Públicas (CEDEX). Recuperado el 20 de 09 de 2013, de: http://www.vitoria-gasteiz.org/wb021/http/contenidosEstaticos/ adjuntos/38988.pdf

Shigiang, W.; Ruixiang, S.; Kun, Y. y Yu, W. (2008). The research of noise induced tympanic membrana perforation in cotton textile industry. IEEE. Conference publications, 1587-1790.

Simón, F., Morales, E., y otros. (20 de 10 de 2008). Caracterización del ruido de la ciudad de Belem. Acustica 2008, Universidad de Coimbra. 1-8. Recuperado el 02 de noviembre de 2013, de: http:// digital.csic.es/bitstream/10261/8157/1/Caracterizacion_ruido_Belem.pdf

Sommerhoff, J.; Recuero, M. y Suárez, E. (2004). Community noise survey of the city of Valdivia, Chile. Applied Acoustics, 65(4), 643-656.

Subroto S. y Dhatrak, S.V. (2008). Occupational noise-induced hearing loss in India. Indian Journal of 
Occupational and Environmental Medicine, 12, 53-56.

Suebsak, N.; Tawan S. y Supphawat, B. (1999). Analytical procedure for constructing noise contours. International Journal of Industrial Ergonomics, 23(1-2), 123-127.

Suter, A.H. (2001). Naturaleza y efectos del ruido. En: J.M. Stellman, M. McCann y L. Warshaw, Enciclopedia de salud y seguridad en el trabajo (pp. 472476). Organización Internacional del Trabajo.
Wu, Q.; Yang, X.; Liu, H. y Dong, H. (2008). Mobile Guardian: A novel positioning and monitoring system for outdoor special users based on GPS. IEEE International Symposium, 560-600.

Ye, O.; Zang, J. y Li, J. (06 de2011). The application and research of data mapping in spatial-relational database. IEEE. Computer Science and Service System (CSSS), 2011 (pp. 791-794).

\section{(c) (1) $(\Theta$

